\title{
Development of a Magnetic Levitation System for Additive Manufacturing: Simulation Analyses
}

\author{
Parichit Kumar, Yuze Huang, Ehsan Toyserkani, Mir Behrad Khamesee Member, IEEE, \\ Department of Mechanical and Mechatronics Engineering, University of Waterloo, Waterloo, Ontario, Canada, \\ N2L $3 G 1$
}

\begin{abstract}
Magnetic levitation has been always a very promising field. Due to its versatility, it has garnered interest in fields energy harvesting, high speed transportation and high precision micro-manipulation amongst other fields. However, it's application within the sphere of Additive Manufacturing (AM) has been negligible. Thus, for the first time, the work conducted here works with the development of a novel technique utilizing magnetic levitation for stable suspension for AM. Current AM technique are heavily dependent on the building the part on a substrate. The substrate limits the use of multiple material nozzles and also requires significant post-processing. The use of the principle of magnetic levitation can bypass the need for this substrate. Primary emphasis is placed on computation of eddy currents and Lorentz force produced from the magnetic levitation system and also on finding the relevant parameters affecting several output parameters like position of levitation, force experienced by the object suspended etc. A temperature analysis is also conducted to ensure that the principles used here are valid. ANSYS Ansoft Maxwell Modules are used to determine these input parameters. The work is subsequently validated through the transient module of the same module. The results presented here depict the viability of the system within the AM environment and the successful elimination of the need for a substrate.
\end{abstract}

Index Terms-Magnetic Levitation, Stable Suspension, Eddy Currents, Additive Manufacturing

\section{INTRODUCTION}

A DDITIVE manufacturing (AM) is the process of manufacturing objects from 3-Dimensional data. AM employs layer by layer addition of material to build the part [1]. There are several types of AM. The work conducted here primarily focuses on the Direct Energy Deposition (DED) technique. Also known as Direct Metal Deposition (DMD) technique, DED is an AM technique that utilizes the concept of simultaneously feeding powder with an energy source (eg. laser) to build the part. This technique also incorporates the principle of layer by layer addition for its successful implementation [2].

DED techniques have several advantages within the AM environment. For instance, DED has a high material deposition rate and high material utilization. In addition, this technique is superior for repair and add-on based applications. This technique can also be used for the installation of wear-resistant materials on products for extending life [3].

The authors are with the Department of Mechanical and Mechatronics Engineering, University of Waterloo, Waterloo, Ontario, Canada, N2L 3G1. Corresponding Author: M.B. Khamesee, khamesee@uwaterloo.ca

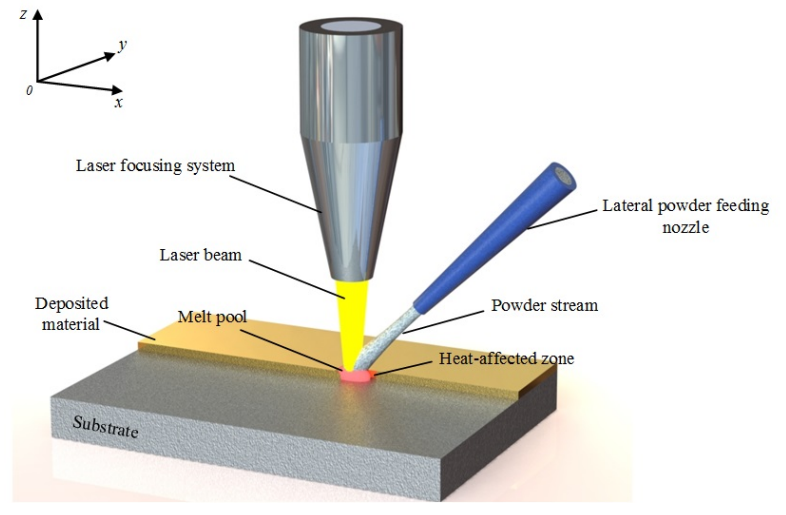

Fig. 1: Conventional Direct Metal Deposition Technique from [4]

As it can be seen in Fig. 1, there is a very strong dependence on the use of a substrate for its successful implementation. The primary function of the substrate is to constrain the article being manufacturing. The part being manufacturing is fused to the substrate to ensure that there is no motion in the $x-y$ plane. The part is subsequently built layer by layer in the $\mathrm{z}$ axis [5]. [5] also studies the build up of residual stresses during the manufacturing process. Due to the constraining feature of the substrate, these stresses cannot be relieved. Thus, the articles being built are vulnerable to fracture. The work shown in this paper aims to bypass the need for this substrate. This is done through the use of magnetic levitation principles to actively levitate the article being manufacturing and producing the necessary restoration forces to stably hold the object.

Fig. 1 depicts a conventional system setup for this technique. It can be seen that the system is constrained to the use of a single nozzle for material deposition. However, by the incorporation of Magnetic Levitation principles, multiple nozzles can be employed to enable faster manufacturing capabilities.

Use of eddy currents to generate forces has been a popular means of generating non-contact forces. [6] studies the use of eddy currents to generate stabilizing restoration forces on tumbling uncontrolled satellites, thereby showing the viability of the principle.

The primary principle of operation is the principle of Electro-dynamic Suspension (EDS) to facilitate stable suspension. EDS has garnered a lot of attention in applications like high speed MagLev Trains. [7] develops a theoretical model and also conducts an experimental study of two magnets 
(HTSC and Halback-arrayed) to determine the eddy currents induced and the subsequent Lorentz force generated. The two are subsequently compared to determine which magnet arrangement produces the maximum levitation force. [8] investigates the use of two Halbach arrays (which are off-set from one another) placed above and below the object to be suspended. The work presented places a strong emphasis on the optimization and subsequent verification of the design. Thus, it can be clearly seen that EDS has garnered significant interest within the academic environment. However, there has been no work presented for the application of this principle within AM environments. [9] is a patent (developed by Boeing Inc.) that revolves around the conceptual idea of a levitation system suitable for AM applications. The description provided in [9] is quite abstract. However, it does reinforce the potential validity of the application of magnetic levitation for AM.

This paper focuses on the calculation of relevant parameters (Peak current and frequency) of AC supply to the magnetic levitation system. The primary objective of the system is to ensure that the solids being levitated achieve stable levitation at a desired height. Multiple cases with added layers are considered for the analysis.

In the work presented here, the emphasis has been placed on simulation-based work, similar to the trend shown in [10] - [13]. [10] shows the development of an analytical model to study effects of pulsed-eddy-currents within nondestructive testing applications. The analytical model is validated through simulations alone. [11] shows the development of a modelling scheme to model transient eddy currents in inhomogeneous mediums. The work is then applied to the FELIX brick experiment. However, no real experimental data was presented to validate the work presented here. [12] deals with the development of hysteresis loses in the cores of a 3 phase transformer. The work develops models for several contributing factors and obtains overall resultant hysteresis loss. However, no experimental work is presented to validate the work. More recently, [13] has presented a simulation study to study the effect of velocity of probes, and subsequently the Lorentz force induced, in defect depth estimation. Following the same trend, the work presented here is primarily validated through simulations run in ANSYS EM Modules. The validity and fidelity of simulations have been shown in Appendix A.

\section{THEORY}

\section{A. Principle of Eddy Current Levitation}

The system utilizes the principle of induced eddy currents for the levitation. According to Faraday's law, a voltage is induced in a conductor loop when it is subjected to a time varying magnetic flux. As a result, a current flow exists in this conductor. This current flow is defined as the eddy currents [14]. These induced eddy currents are described best through Maxwell's equations, shown in Eqn. 1 - 2 [15].

$$
\begin{gathered}
\nabla \times E=\nabla \times \frac{J}{\sigma}=-\frac{\partial B}{\partial t} \\
\nabla \times H=J
\end{gathered}
$$

where $\mathrm{E}$ is the induced electric potential, $\mathrm{J}$ is the induced currents, $\sigma$ is the conductivity of the material, $\mathrm{H}$ is the magnetic field strength and B is the magnetic flux density of the source.

The levitator setup consists of two cylindrical coils carrying time-varying sinusoidal current. The coil closer to the centre (i.e. the primary coil) is used for the generation of the primary vertical force. The coil closer to the outer edge (i.e. the secondary coil) is utilized for generation of stabilizing lateral forces. These coils are embedded within a ferrite core block. The various dimensions of the system are obtained through isolated optimization of the parameters. The schematic for the levitator setup are shown in the Fig. 2.

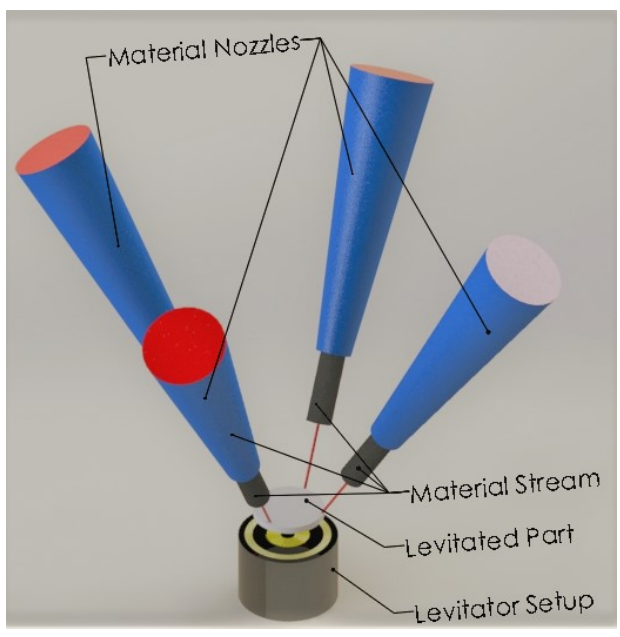

Fig. 2: Schematic of Optimized Levitator

The technique presented has several significant advantages over conventional techniques. The levitated part serves as an initial base for building the part. Ideally, the base would end up being a part of the final component. Fig. 2 shows the use of multiple material jets to build the part. This idea is further supported by the research presented in [9]. Thus, the system allows for multi-directional deposition, thereby improving the efficiency of building the part.

In addition to the use of multiple material jets, the levitator setup is also capable of producing the torque necessary to flip

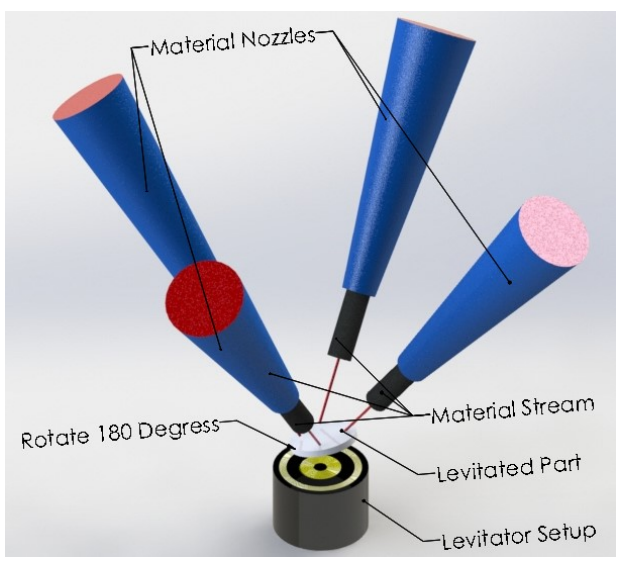

Fig. 3: Rotational Ability of Levitator 
the levitated part 180 degrees without the need for external force application. This leads to the availability of both sides of the initially levitated part for additive manufacturing operations. This is shown in fig. 3 .

For the initial phase of the research, the emphasis is placed on the building of parts with simple geometries. In addition, the levitator setup is thought of as an add-on item to an existing additive manufacturing machine. The methodology of use is that the levitator setup would be placed in place of the substrate. The primary advantage of the system would be in effect when the additive manufacturing system consists of multiple material nozzles working together to accelerate the manufacturing operations. Thus, the levitator setup can be thought of as an initial investment from a cost perspective.

1) Vertical Force for Stable Levitation: The induced eddy currents interact with the primary time-varying magnetic field. According to the principle of Lorentz force, there is a force generated through the interactions of the magnetic fields of the induced eddy currents with the magnetic fields of the coil. This results in the generation of a force that results in the stable suspension of the solid. The Lorentz force is defined by Eqn. 3 [16].

$$
F=\iiint_{V} J \times B d V
$$

As shown in Eqn. 3, the vertical levitation force is a function of the induced eddy currents and the magnetic field of the primary coil setup. Both of these parameters are primarily dependent on the magnitude of the applied current (peak current) and the frequency of the alternating current supply.

2) Position Relative to the Levitator: In order to conduct AM operations, it is necessary to gain positional control of the suspended object. In conventional methods, the substrate provides the relevant constraining forces necessary to prevent motion during the operation. Thus, it is necessary to hold the object at a desired height with stability. Since the stability in the vertical direction is primary dependent on the vertical force imposed on the object, the position of the suspended object is also dependent on the magnitude of the applied current and the associated frequency of the alternating current supplied to the coils.

3) Temperature Increase due to Induced Eddy Currents: According to [17], there is significant amount of heat energy generated due to the induced eddy currents. This heat energy serves as the primary principle for the implementation of induction heating. These heat loses can be directly attributed to the internal resistance of the object suspended. Thus, computation of the temperature as a function of time are relevant.

According to [15], for a coil operating for extended periods, a static thermal model is satisfactory for the analysis. Conduction, convection and radiation are the primary modes of heat transfer. The governing equations for these are shown in Eqn. $4-6$.

Using these equations, the output thermal characteristics of the solid can be determined.

$$
h_{r}=\frac{\sigma_{t} \epsilon\left(T_{s}^{4}-T_{a}^{4}\right)}{T_{s}-T_{a m}}
$$

$$
\begin{gathered}
Q=\left(h_{c}+h_{r}\right) \cdot A \cdot\left(T_{s}-T_{a}\right) \\
T=\frac{Q}{C_{\text {thermal }}}
\end{gathered}
$$

where $h_{c}$ is the free convection heat transfer coefficient, $h_{r}$ is the radiation heat transfer coefficient, $\sigma_{t}$ is the Stefan Boltzman constant, $\epsilon$ is the emmisivity of Aluminum, $C_{\text {thermal }}$ is the specific heat capacity of the solid suspended.

\section{B. Levitation Ability of Materials}

Use of magnetic levitation for stable suspension requires the fulfillment of certain criteria. According to [18], the parameter for consideration of the levitation ability of a material is the ratio of the maximum levitation force experienced by the object suspended to the weight of the object. That is

$$
\text { LevAbility }=\frac{F_{\max }}{W}
$$

where $F_{\max }$ is the maximum levitation force produced by the coil setup, $\mathrm{W}$ is the weight of the object suspended.

Due to the dependence of the system on induced eddy currents in the presence of a time-varying magnetic fields (assumed to sinusoidal), the levitation capability is directly proportional to the conductivity of the material. This is depicted by the Eqn. 8 - 10.

$$
\begin{gathered}
E_{\text {induced }}=\frac{d \Phi}{d t} \\
J=E_{\text {induced }} \sigma \\
F_{\text {lev }}=\int J \times B d V
\end{gathered}
$$

Here, $\phi$ is the magnetic flux, $\mathrm{J}$ is the induced eddy currents and $\sigma$ is the conductivity of the material. Thus, in order to maximize the levitation force experienced by the levitated object, the only material property for consideration is the conductivity of the material.

In addition, the levitation ability of a material is dependent on the weight of the body suspended. Thus, the levitation capability of the material is inversely proportional to the density of the material. Accordingly, the parameter of relevance for consideration of the levitation capability of a material is given by Eqn. 11 .

$$
\text { LevAbility }=\frac{F_{\max }}{W}=\frac{\sigma}{\rho}
$$

where $\sigma$ is the electrical conductivity of the material and $\rho$ is the density of the material. 


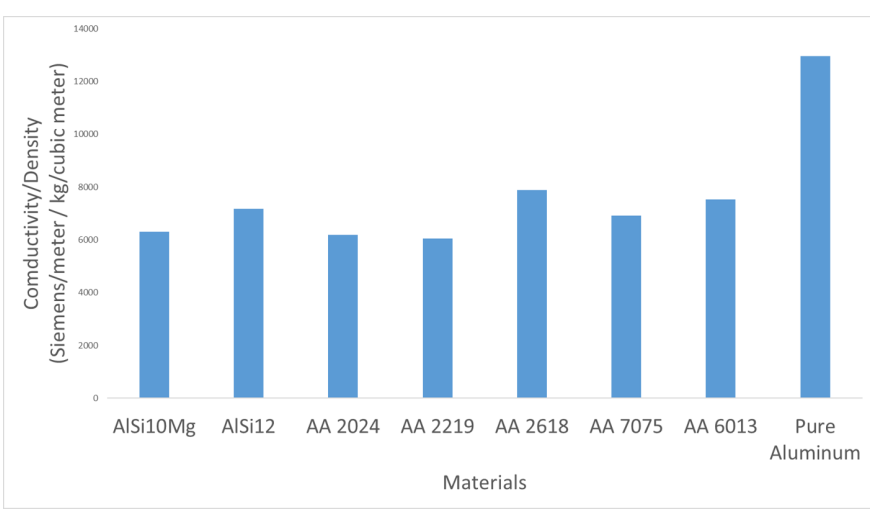

Fig. 4: Levitation Ability of Aluminum Alloys

\section{Aluminum Alloys for Analysis}

Due to the incorporation of principles of electrodynamic levitation, the system is restricted to use non-ferrous materials. Due to the magnetization ability of ferrous materials, they are not ideal for this application. However, through literature review, it is found that most AM applications employ the use of Aluminum alloys.

Based on [19] and [20], some of the aluminum alloys with applications within the AM environment were noted. The application of these alloys within the AM environment are shown in TABLE I. In order to determine the viability of these alloys within the magnetic levitation application, their levitation capability ratios are plotted and compared. This is depicted in the bar graph shown Fig. 4. Pure Aluminum, which is documented to have a high levitation ability [18], is added as a reference point. As it can be seen, the levitation ability of most alloys are comparable to Aluminum. Thus, these alloys are appropriate for AM Applications.

TABLE I: Applications of Alloys within the AM Environment

\begin{tabular}{|l|l|}
\hline Material & Application \\
\hline AlSi10Mg & $\begin{array}{l}\text { automotive, aerospace and } \\
\text { domestic industries [21] }\end{array}$ \\
\hline AlSi12 & $\begin{array}{l}\text { aerospace, automotive and } \\
\text { biomedical applications. [22] }\end{array}$ \\
\hline AA 2024 & $\begin{array}{l}\text { Used in the production of commercial } \\
\text { and military aircrafts. [23] }\end{array}$ \\
\hline AA $2219(\mathrm{AlCu} 6 \mathrm{Mn})$ & $\begin{array}{l}\text { mechanically demanding } \\
\text { applications in light-weight } \\
\text { design. [24] }\end{array}$ \\
\hline AA 6013 & $\begin{array}{l}\text { corrosion resistance based } \\
\text { applications [23] }\end{array}$ \\
\hline AA $2618(\mathrm{AlCu} 2 \mathrm{Mg} 1.5 \mathrm{Ni})$ & $\begin{array}{l}\text { mechanically demanding } \\
\text { applications in light-weight } \\
\text { design. [24] }\end{array}$ \\
\hline AA 7075 & $\begin{array}{l}\text { Used for fuselage skins, stringers, } \\
\text { and bulkheads, as well as for } \\
\text { wing skins, panels, and covers. [23] }\end{array}$ \\
\hline
\end{tabular}

\section{Simulations AND Results}

\section{A. Test Cases for Analysis}

The peak current and the frequency of the alternating current supplied are the key parameters driving the magnitude and direction of the levitation force generated. ANSYS EM Suite - Maxwell 2D has been used to determine these parameters.
TABLE II: Specifications of the Three Cases considered

\begin{tabular}{|c|c|c|c|}
\hline Case & Base Plate & Layer 1 & Layer 2 \\
\hline Case 1 & $\begin{array}{l}\mathrm{r}=25 \mathrm{~mm} \\
\mathrm{~h}=5 \mathrm{~mm} \\
\text { Mass }=0.026 \mathrm{~kg}\end{array}$ & - & - \\
\hline Case 2 & $\begin{array}{l}\mathrm{r}=25 \mathrm{~mm} \\
\mathrm{~h}=5 \mathrm{~mm} \\
\text { Mass }=0.026 \mathrm{~kg}\end{array}$ & $\begin{array}{l}\mathrm{r}=20 \mathrm{~mm} \\
\mathrm{~h}=5 \mathrm{~mm} \\
\text { Mass }=0.0047 \mathrm{~kg}\end{array}$ & - \\
\hline Case 3 & $\begin{array}{l}\mathrm{r}=25 \mathrm{~mm} \\
\mathrm{~h}=5 \mathrm{~mm} \\
\text { Mass }=0.026 \mathrm{~kg}\end{array}$ & $\begin{array}{l}\mathrm{r}=20 \mathrm{~mm} \\
\mathrm{~h}=5 \mathrm{~mm} \\
\text { Mass }=0.0047 \mathrm{~kg}\end{array}$ & $\begin{array}{l}\mathrm{r}=5 \mathrm{~mm} \\
\mathrm{~h}=5 \mathrm{~mm} \\
\text { Mass }=0.0011 \mathrm{~kg}\end{array}$ \\
\hline
\end{tabular}

For the purpose of this analysis, three separate cases were considered. Some specifications for the system are shown in table II. It should be noted that the solids suspended are composed of AlSi12.

\section{B. Calculation of Lift Current}

First, the emphasis is placed on the calculation of the lift current. The lift current is defined as the minimum peak current required to stably levitate the objects (details in TABLE II) at specific frequencies $(800-1000 \mathrm{~Hz})$. The analysis is done to calculate the peak current $8 \mathrm{~mm}$ and $15 \mathrm{~mm}$ away from the levitator. The calculation is conducted through the use of the principle of least square fitting.

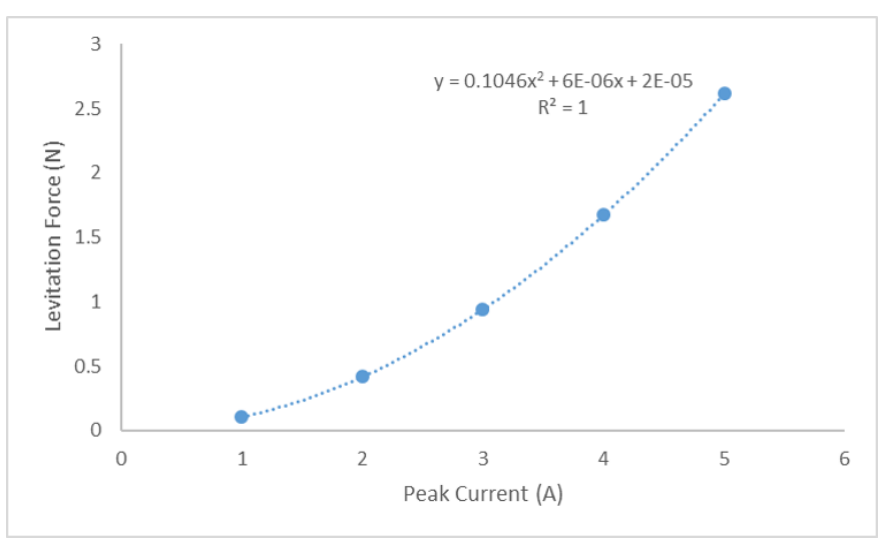

Fig. 5: Peak Current vs Levitation Force

In order to do this, a plot depicting the relationship between the magnitude of current of the AC Supply and the zcomponent of the levitation force was developed. This was obtained by documenting the levitation force for various current inputs for an alternating current supply. The levitation force was documented for the various peak currents at $1000 \mathrm{~Hz}$ frequency. Through least square fitting, the equation of curve was obtained. This equation was then used to interpolate the required peak current for stable levitation. The required force (i.e. the weight of the disk suspended) was given a tolerance of $10 \%$. The plot is shown in Fig. 5.

Finally, the fit equation was equated to the gravitational force of the mass to be suspended, as shown in Eqn. 12. Solving, the 'lift' current, i.e., the current required to be supplied to the current carrying coil to stably levitate the mass is obtained. This is shown in the equation 12. As shown in Fig. 5, the coefficient of determination is 1 . This shows that 
the least square fitting equation represents the behavior of the system quite strongly.

$$
\begin{array}{r}
\text { LevForce }=0.1405 I^{2}+0.000006 I+0.00002 \\
0.026 \times 9.81=0.1405 I^{2}+0.000006 I+0.00002 \\
I=1.636 A
\end{array}
$$

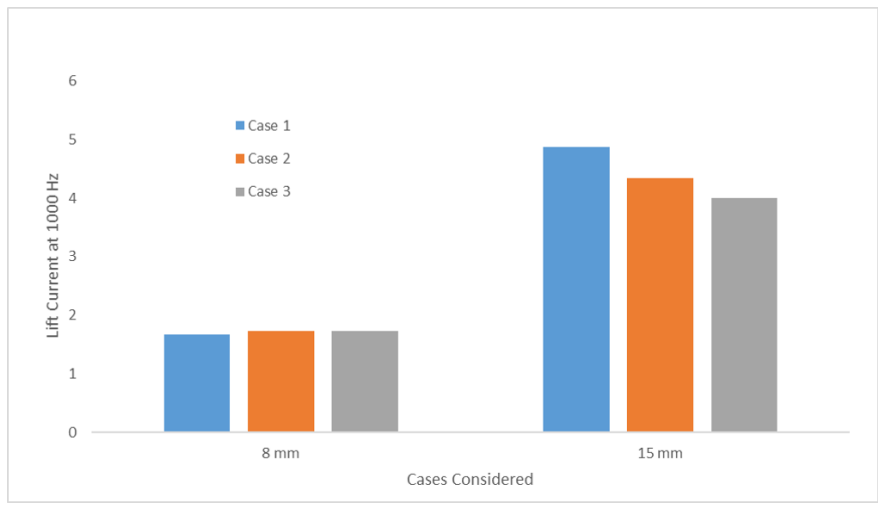

Fig. 6: Peak Lift Current Calculated for $1000 \mathrm{~Hz}$ Supply for all cases

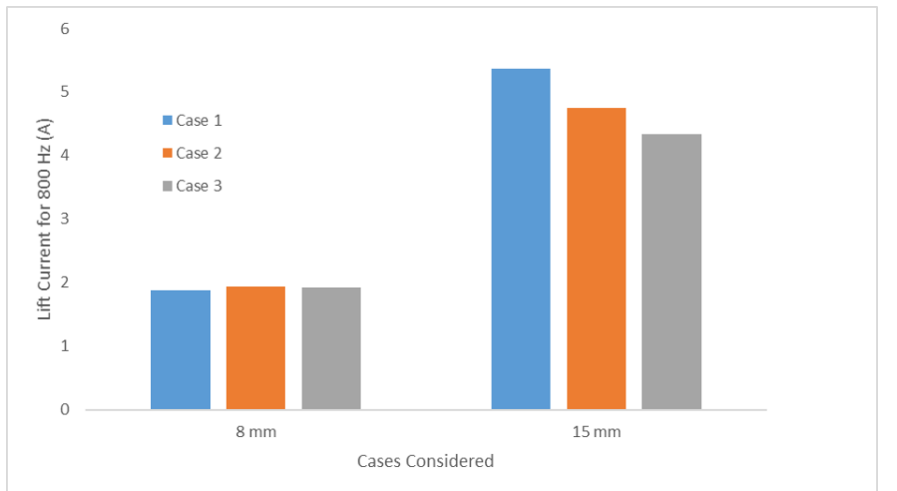

Fig. 7: Calculated Lift Peak Current of AC Current for 800 $\mathrm{Hz}$ Frequency

The process was repeated for all cases for $1000 \mathrm{~Hz}$ and 800 $\mathrm{Hz}$ situations. The results are shown in Fig. 6 and 7.

\section{Calculation of Lift Frequency}

Next, the emphasis was placed on the calculation of the lift frequency. The lift frequency of the AC supply is defined as the minimum frequency required to stably levitate the solids (Table II) at specific peak currents (4 \& 5 A). The calculation of the peak frequencies are also conducted using least squared fitting. Similar to the analysis shown for the calculation of the lift current, a plot of Frequency vs Levitation force is developed and used to develop the least squared fitting equation. The analysis is done for all cases at $8 \mathrm{~mm}$ and 15 $\mathrm{mm}$ away from the levitator. The results are shown in figure 8 and 9.

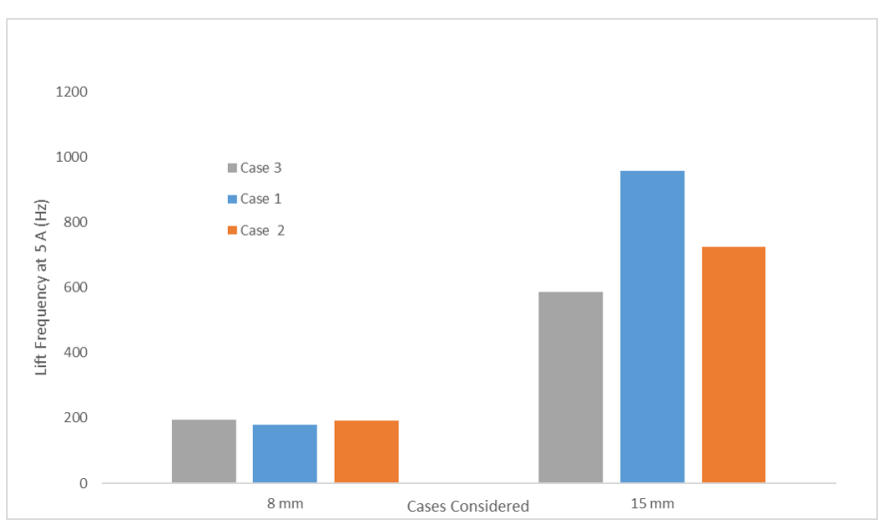

Fig. 8: Calculated Lift Frequency of AC Current for 5 A Peak Current

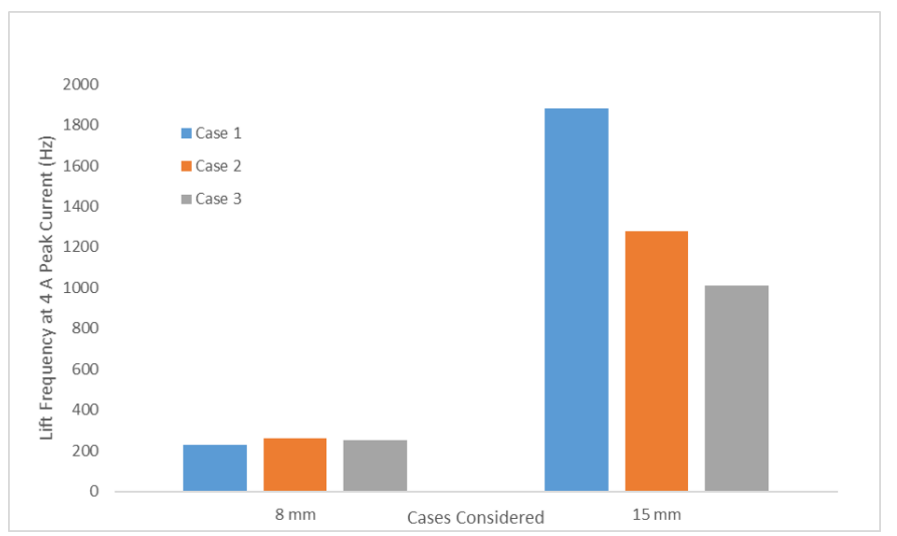

Fig. 9: Calculated Lift Frequency of AC Current for 4 A Peak Current

\section{VALIDATION}

In an attempt to verify the calculated currents and frequencies, an analysis was conducted in the transient domain. The Transient Module for ANSYS Maxwell 2D was used. The module provides the capability to observe the variation of several parameters (like Force, position, velocity etc.) as a function of time. ANSYS 2D module was used to ease meshing and thereby reduce computation time.

The primary objective of the analysis here is to show that the lift current calculated here was sufficient for the stable levitation of the solid. For the purpose of this verification, Case 1 (Table II) was used. The mass of the solid suspended is 0.026 $\mathrm{kg}$. The alternating current supplied to the levitator is shown in Fig. 10. It shows the characteristics of the input current (1.636 A Peak Current, $1000 \mathrm{~Hz}$ Frequency). The current calculated stably levitates the solid at $8 \mathrm{~mm}$ above the levitator setup.

\section{A. Parameters vs Time}

The simulations are conducted based on the model described. The primary parameters that need to observed to validate the model is the position of the solid as a function of time. Ideally, the solid would rise to the desired height ( $8 \mathrm{~mm}$ above levitator) and stay there. However, due to the sinusoidal nature of the forces produced, slight oscillations 


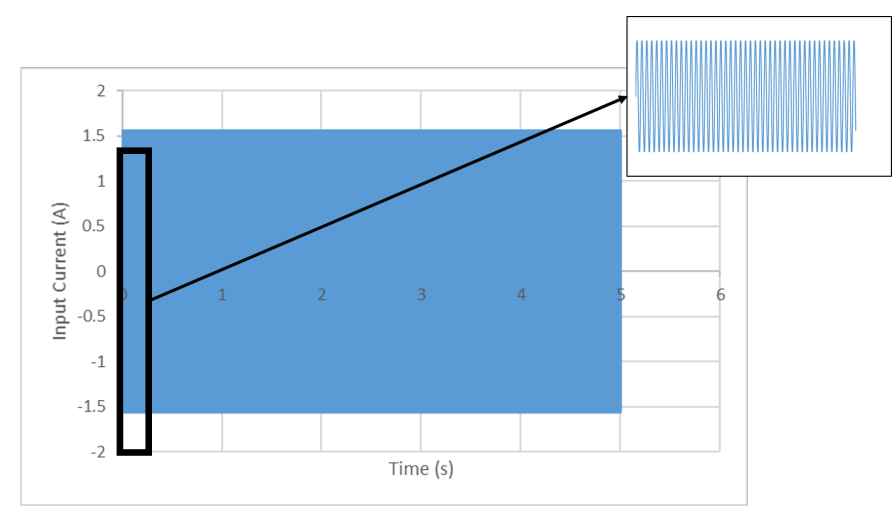

Fig. 10: Input Current Supplied to the Levitator

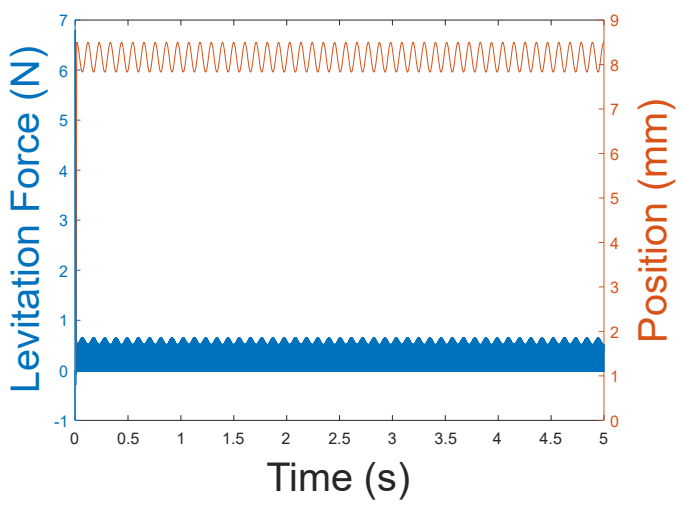

Fig. 11: Position and Levitation Force of the Disc vs Time

are acceptable. The plots for the position of the solid as a function of time (see Fig. 11) and the vertical levitation force (see Fig. 11) are obtained.

Fig. 11 shows that the variation of the position in the $\mathrm{z}$ axis (z) of the solid is between $7.8 \mathrm{~mm}$ to $8.5 \mathrm{~mm}$. These oscillations are relatively small. Thus, the stable levitation at the desired height is achieved. Following an initial transient stage, the levitation force also achieves a steady-state sinusoidal characteristic. Thus, the system analysis shows that the lift current for the solid is validated. The analysis is repeated for all other cases and the results follow the same trend.

\section{B. Temperature vs Time}

The method discussed here depends on the generation of eddy currents. Due to the production of these currents, there are some heat loses within the solid being levitated. Thus, it is crucial to conduct a thermal analysis on the system to ensure that the material stably levitated does not heat up excessively too quickly. Thus, in order to conduct this analysis, ANSYS transient thermal module was used.

The induced eddy currents primarily exist within a characteristic length known as the skin depth $(\delta)$ [15]. The induced eddy currents that exist beyond this characteristic length are very small, and thereby can be ignored. The skin depth is a function of the frequency, permeability and density of the materials. For the test cases discussed here, the $\delta$ value computed is shown in Eqn. 13. Since the currents are produced within this depth, the heat loses originate within this band.

$$
\delta=\sqrt{\frac{\rho}{\pi f_{0} \mu_{r} \mu_{0}}}=2.593 \mathrm{~mm}
$$

where $f_{0}$ is frequency of operation, $m u_{r}$ is the relative permeability and $\mu_{0}$ is the permeability of free space

In order to depict the sustainability of the model, the maximum value of the heat loses produced amongst all nodal points (extracted from the ANSYS EM Maxwell module) are uniformly fed to all nodal points within the skin depth of the disc. This ensures that the temperature estimated is higher than the actual temperature distribution of the disc. The primary objective of this estimation technique is to ensure that even in relatively harsh circumstances, the system can be feasible. For the analysis, the ambient temperature is assumed to be $22^{\circ} \mathrm{C}$.

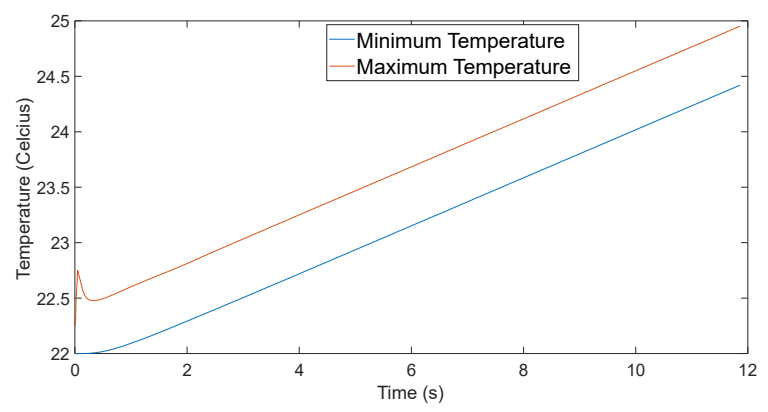

Fig. 12: Minimum and Maximum Temperature of the Solid vs Time

Fig. 12 shows the variation of the temperature of the solid as a function of time. The analysis is conducted for 12 seconds here. As it can be seen, the maximum temperature increase after 12 seconds is about $4^{\circ} \mathrm{C}$. Using least squared fitting, the time required to heat the system to the Melting Point of AlSi12 $\left(660^{\circ} \mathrm{C}\right)$ is computed. The fitted curve for the temperature vs time function is shown in Fig. 13. As it can be seen, the melting point of the solid is achieved after 2850 seconds. Thus, with external cooling, the stable levitation can be achieved for extended periods without melting the solid being suspended.

\section{CONClusion}

This paper presents the work associated with the stable levitation of metallic solids through the principle of electrodynamic levitation. The paper primarily focuses on the use Aluminum Alloys suitable for AM applications. The analysis is conducted using AlSi12. However, since the levitation ability of all alloys shown are within close range, the system and principles are valid for these as well. The primary emphasis is placed on the calculation of the peak current and frequency calculations of the alternating current supply fed to the optimized levitator setup. The computed parameters are then validated based on the position of the solid at steady state, the force experienced by the solid at steady state and the temperature increase of the solid. Through the analysis, it can be clearly concluded that the system produced can stably levitate Aluminum Alloys and maintain the stable levitation 


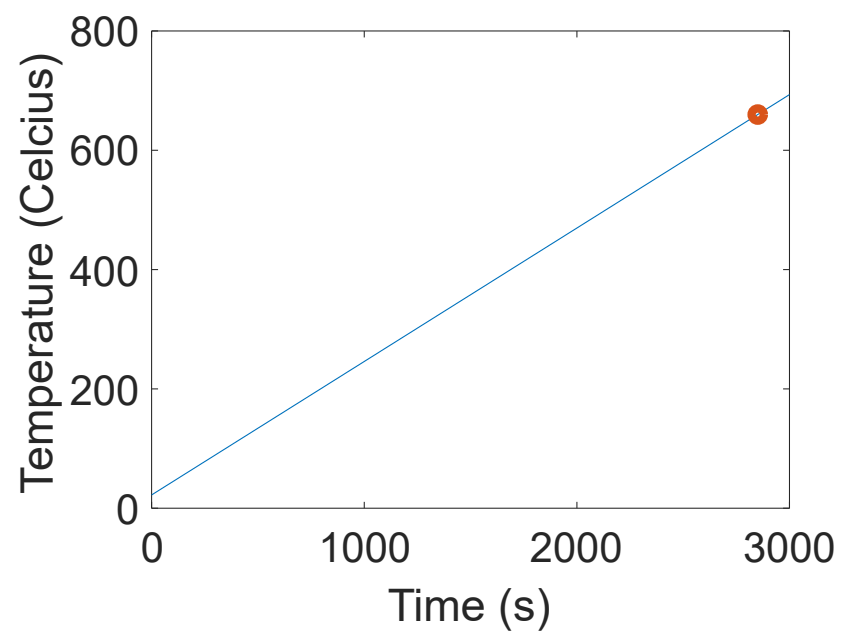

Fig. 13: Fitted Curve (from Maximum Current) for Temperature vs Time with Melting point of $\mathrm{Al}\left(660^{\circ}\right)$ plotted

for extended periods of time. A significant limitation of the methodology presented here is that the technique is only applicable to electrical conducting materials. As stated in section II-B, the levitation ability of the part is dependent on the electrical conductivity of the material. However, it can be concluded that the system has found to be applicable within the AM environment using metallic alloys (e.g., AlSi12) to build the part. There is a strong dependence on the use of simulations through ANSYS Maxwell. However, given the high fidelity of the simulation results and the reliability of these simulations (depicted in Appendix A), the results presented are well grounded. Further experiments are to be conducted to validate the work presented through experimental data.

\section{APPENDIX A \\ Fidelity OF Simulations THROUGH ANSYS}

ANSYS Maxwell 2D and 3D software has played a very major role in the analysis presented. ANSYS Maxwell 3D Eddy Current Module has been used to calculate the magnitude and frequency of alternating current required for stable suspension. ANSYS Maxwell 3d - Transient Module was used to generate the plots as a function of time.

The simulation results are quite high fidelity and have been proven to be very reliable in terms of mimicking real world performance of magnetic systems. For instance, the system presented in [15] was simulated in ANSYS Maxwell and the results of the simulation was compared to the results of the paper.

[15] presents the levitation of a coil over an Aluminum disc. The system consists of a single coil with 107 turns. The coil has a radial width of $2 \mathrm{~mm}$ (outer diameter $=5 \mathrm{~mm}$ and inner diameter $=3 \mathrm{~mm}$ ) and an axial height of $1.6 \mathrm{~mm}$. The dimension of the disc is much larger than the coil (treated as infinite). The disc was taken to have a radius of $500 \mathrm{~mm}$ and height of $10 \mathrm{~mm}$. According to [15], it was determined experimentally that the current required to levitate the coil at
$20 \mathrm{~mm}$ was $39 \mathrm{~A}$ at a frequency of $60 \mathrm{~Hz}$. The results obtained from the simulations are presented in fig. 14 .

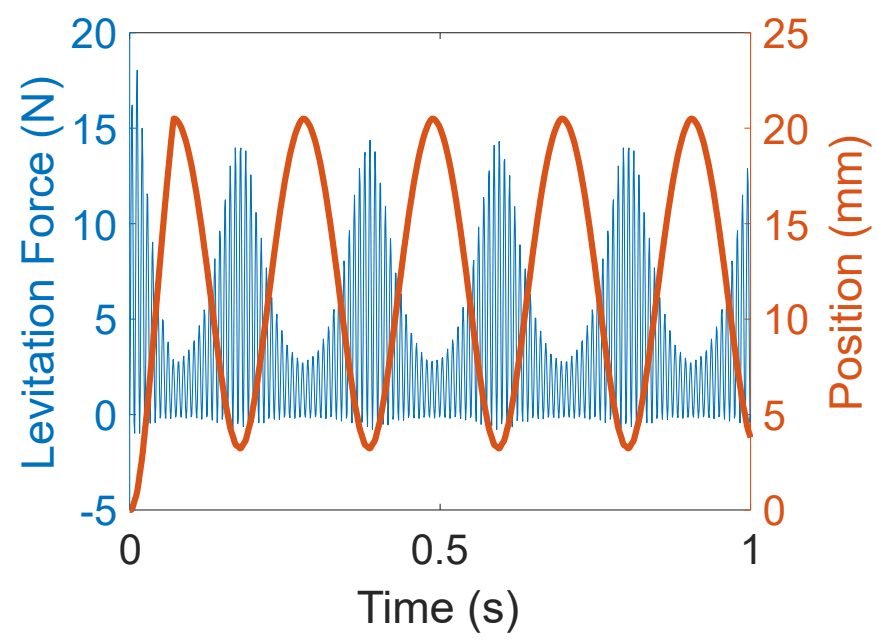

Fig. 14: Resulting plots from Simulations for the system studied in [15]

As it can be noted, the results of the simulations are quite similar to the experimental results presented. The coil levitates at $20.5 \mathrm{~mm}$. Thus, the results obtained from the simulation are within $2.5 \%$ of the experimental data presented. Thus, it can be concluded that the simulations have a very high fidelity, thereby provide reliable data.

\section{REFERENCES}

[1] Frazier, W.E. J. of Materi Eng and Perform (2014) 23: 1917. https://doi.org/10.1007/s11665-014-0958-z

[2] A. Saboori, D. Gallo, S. Biamino, P. Fino, and M. Lombardi, "An Overview of Additive Manufacturing of Titanium Components by Directed Energy Deposition: Microstructure and Mechanical Properties," Applied Sciences, vol. 7, no. 9, p. 883, 2017.

[3] J.-Y. Lee, J. An, and C. K. Chua, "Fundamentals and applications of 3D printing for novel materials," Applied Materials Today, vol. 7, pp. 120-133, 2017.

[4] Y. Huang, M. Ansari, H. Asgari, M. H. Farshidianfar, D. Sarker, M. B. Khamesee, and E. Toyserkani, "Rapid prediction of real-time thermal characteristics, solidification parameters and microstructure in laser directed energy deposition (powder-fed additive manufacturing)," Journal of Materials Processing Technology, vol. 274, p. 116286, 2019.

[5] Jonathan Meyer, "Substrate for additive manufacturing", 14-Aug-2018

[6] X. Liu, Y. Lu, Q. Zhang, and K. Zhang, "An Application of Eddy Current Effect on the Active Detumble of Uncontrolled Satellite With Tilt Air Gap," IEEE Transactions on Magnetics, vol. 55, no. 12, pp. 1-11, 2019.

[7] H. Cho, D. K. Bae, H.-K. Sung, and J. Lee, "Experimental Study on the Electrodynamic Suspension System With HTSC and PM Halbach Array Magnets," IEEE Transactions on Applied Superconductivity, vol. 18, no. 2, pp. 808-811, 2008.

[8] Duan, Jiaheng, et al. "Analysis and Optimization of Asymmetrical Double-Sided Electrodynamic Suspension Devices." IEEE Transactions on Magnetics, vol. 55, no. 6, 2019, pp. 1-5., doi:10.1109/tmag.2019.2894709.

[9] W. A. Harkness and J. H. Goldschmid, "Free-Form Spatial 3-D Printing Using Part Levitation," 04-Feb-2016.

[10] X.-W. Dai, R. Ludwig, and R. Palanisamy, "Numerical simulation of pulsed eddy-current nondestructive testing phenomena," IEEE Transactions on Magnetics, vol. 26, no. 6, pp. 3089-3096, 1990.

[11] P. Leonard and D. Rodger, "Finite element scheme for transient 3D eddy currents," IEEE Transactions on Magnetics, vol. 24, no. 1, pp. 90-93, 1988. 
[12] E. N.- Juszczak, R. Grzybowski, and J. Brudny, "Modelling of losses due to eddy currents and hysteresis in converter transformer cores during failure," IEEE Transactions on Magnetics, vol. 31, no. 3, pp. 1718-1721, 1995.

[13] Dolker, Eva-Maria, et al. "Velocity-Dependent Lorentz Force Evaluation: A Simulation Study." IEEE Transactions on Magnetics, vol. 55, no. 6, 2019, pp. 1-4., doi:10.1109/tmag.2019.2901579.

[14] E. Kriezis, T. Tsiboukis, S. Panas, and J. Tegopoulos, "Eddy currents: theory and applications," Proceedings of the IEEE, vol. 80, no. 10, pp. 1559-1589, 1992.

[15] M. Thompson, "Electrodynamic magnetic suspension-models, scaling laws, and experimental results," IEEE Transactions on Education, vol. 43, no. 3, pp. 336-342, 2000.

[16] D. J. Griffiths, Introduction to electrodynamics. Cambridge, United Kingdom: Cambridge University Press, 2018.

[17] Fisk M. (2014) Induction Heating. In: Hetnarski R.B. (eds) Encyclopedia of Thermal Stresses. Springer, Dordrecht

[18] Nan, Wang, et al. "Physical Characteristics of Electromagnetic Levitation Processing." Acta Physica Sinica (Overseas Edition), vol. 8, no. 7, 1999, pp. 503-513., doi:10.1088/1004-423x/8/7/005.

[19] N. T. Aboulkhair, M. Simonelli, L. Parry, I. Ashcroft, C. Tuck, and R. Hague, "3D printing of Aluminium alloys: Additive Manufacturing of Aluminium alloys using selective laser melting," Progress in Materials Science, vol. 106, p. 100578, 2019.

[20] Singh, Amrinder, et al. "Direct Laser Metal Deposition of Eutectic Al-Si Alloy for Automotive Applications." TMS 2017 146th Annual Meeting Exhibition Supplemental Proceedings The Minerals, Metals \& Materials Series, 2017, pp. 71-80., doi:10.1007/978-3-319-51493-2_8.

[21] K. Kempen, L. Thijs, J. V. Humbeeck, and J.-P. Kruth, "Mechanical Properties of AlSi10Mg Produced by Selective Laser Melting," Physics Procedia, vol. 39, pp. 439-446, 2012.

[22] S. Siddique, M. Imran, E. Wycisk, C. Emmelmann, and F. Walther, "Fatigue Assessment of Laser Additive Manufactured AlSi12 Eutectic Alloy in the Very High Cycle Fatigue (VHCF) Range up to $1 \mathrm{E} 9$ cycles," Materials Today: Proceedings, vol. 3, no. 9, pp. 2853-2860, 2016.

[23] J.-P. Immarigeon, R. Holt, A. Koul, L. Zhao, W. Wallace, and J. Beddoes, "Lightweight materials for aircraft applications," Materials Characterization, vol. 35, no. 1, pp. 41-67, 1995.

[24] B. Ahuja, M. Karg, K. Y. Nagulin, and M. Schmidt, "Fabrication and Characterization of High Strength Al-Cu Alloys Processed Using Laser Beam Melting in Metal Powder Bed," Physics Procedia, vol. 56, pp. 135-146, 2014. 